\title{
Extracellular Vesicles from Red Blood Cell Products Induce a Strong Pro-Inflammatory Host Response, Dependent on Both Numbers and Storage Duration
}

\author{
Marleen Straat ${ }^{\mathrm{a}, \mathrm{b}} \quad$ Anita N. Böing ${ }^{\mathrm{c}} \quad$ Anita Tuip-De Boer ${ }^{\mathrm{b}} \quad$ Rienk Nieuwland $^{\mathrm{c}}$ \\ Nicole P. Juffermans ${ }^{a, b}$ \\ a Department of Intensive Care Medicine, Academic Medical Center, Amsterdam, The Netherlands; \\ ${ }^{b}$ Laboratory of Experimental Intensive Care and Anesthesiology, Academic Medical Center, Amsterdam, The Netherlands; \\ ${ }^{c}$ Laboratory of Experimental Clinical Chemistry, Academic Medical Center, Amsterdam, The Netherlands
}

\section{Keywords}

Red blood cell units - Extracellular vesicles .

Host response $\cdot$ Cytokines

\section{Summary}

Background: Transfusion of red blood cells (RBCs) is associated with adverse outcome, but the causative factor is unknown. Extracellular vesicles (EVs) have pro-inflammatory properties. We hypothesized that EVs released from both fresh and stored RBC products can induce a host inflammatory response in a dose-dependent manner. Methods: Whole blood was incubated with supernatant from RBC units stored for different time periods, either containing (different numbers of) EVs or depleted from EVs. Results: Incubation with both fresh and stored supernatant containing EVs induced a strong host response with production of TNF, IL-6 and IL-8. In supernatant depleted from EVs, this host response was completely abrogated. IL-10 levels were not affected. EV-induced host response was both dependent on the number of EVs as well as on storage time. Conclusions: EVs from both fresh and stored RBC units illicit a strong inflammatory host response in recipients and may therefore contribute to adverse outcome of RBC transfusion.

(C) 2015 S. Karger GmbH, Freiburg

\section{Introduction}

Despite having obvious benefits, red blood cell (RBC) transfusion also carries risks. In several patient populations, RBC transfusions have been associated with inflammatory conditions such as acute lung injury, nosocomial infections, and systemic inflammatory response syndrome [1-3]. The mechanism of transfusion-related adverse effects is unknown. Experimental studies suggest that $\mathrm{RBC}$ storage duration is a risk factor for the occurrence of transfusion-related adverse events $[4,5]$, although results of clinical studies on the influence of age of RBCs on outcome are conflicting [58]. Previously, we found that the supernatant of a RBC product, but not the RBCs themselves, induce pulmonary inflammation in a rodent model, but the causative factor was not identified [5].

Extracellular vesicles (EVs) are small phospholipid vesicles released from a wide variety of cells, including leukocytes, platelets, RBCs and endothelial cells. EVs have also been referred to as microparticles. EVs facilitate intercellular exchange of receptors, ligands and signaling molecules, without direct cell-to-cell contact [9]. High concentrations of RBC-derived EVs are present in the supernatant of RBC transfusion bags [10]. These EVs have pro-coagulant properties, including thrombin generation and activation of platelets. During storage, both the concentration of EVs as well as their pro-coagulant properties increase $[11,12]$.

We hypothesize that EVs accumulating in $\mathrm{RBC}$ products during storage contribute to a pro-inflammatory host response in recipients, which is related both to their amount as well as to the storage duration.

\section{Material and Methods}

\section{Preparation of Samples}

Samples were collected from RBC units that were transfused to patients. These RBC units were prepared and stored according to Sanquin blood bank standards, including leukoreduction. Directly after transfusion, $3 \mathrm{ml}$ of blood product that were left behind in the bag were 1:1 diluted with filtered phosphate-buffered saline (PBS). The diluted RBC sample was centrifuged for 20 $\min$ at $1,550 \times g, 20^{\circ} \mathrm{C}$ to remove RBCs. Subsequently, the EV-containing su-

\section{KARGER \\ Fax +497614520714

\section{(c) 2015 S. Karger GmbH, Freiburg}

1660-3796/15/0434-0302\$39.50/0 

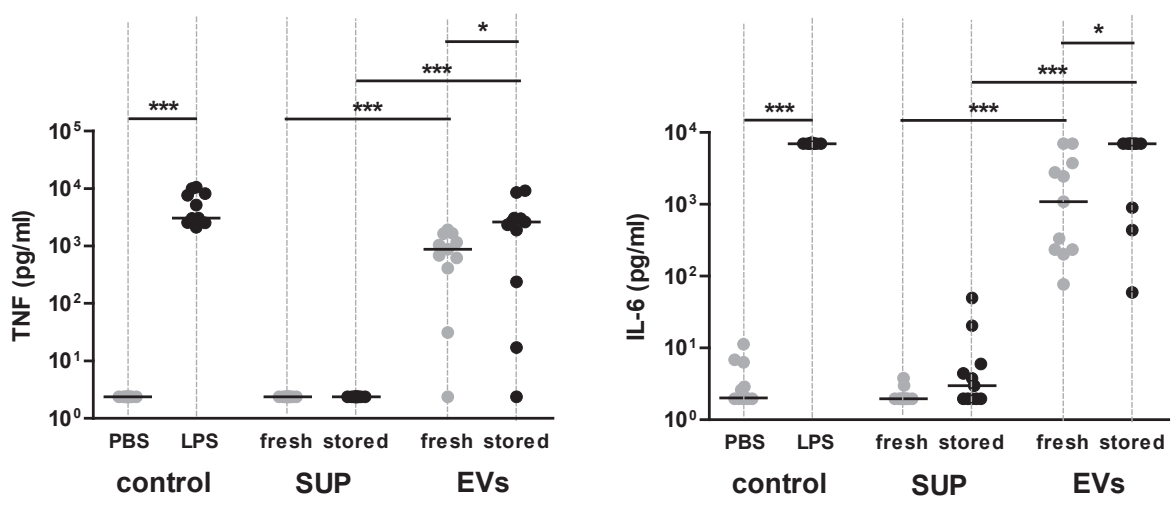

Fig. 1. Whole blood stimulated with a fixed volume of supernatant (sup) derived from fresh and stored RBC bags either containing EVs or depleted from EVs $\left(\mathrm{n}=11\right.$ per group). ${ }^{*} \mathrm{p}<0.05,{ }^{* * *} \mathrm{p}<0.0001$.
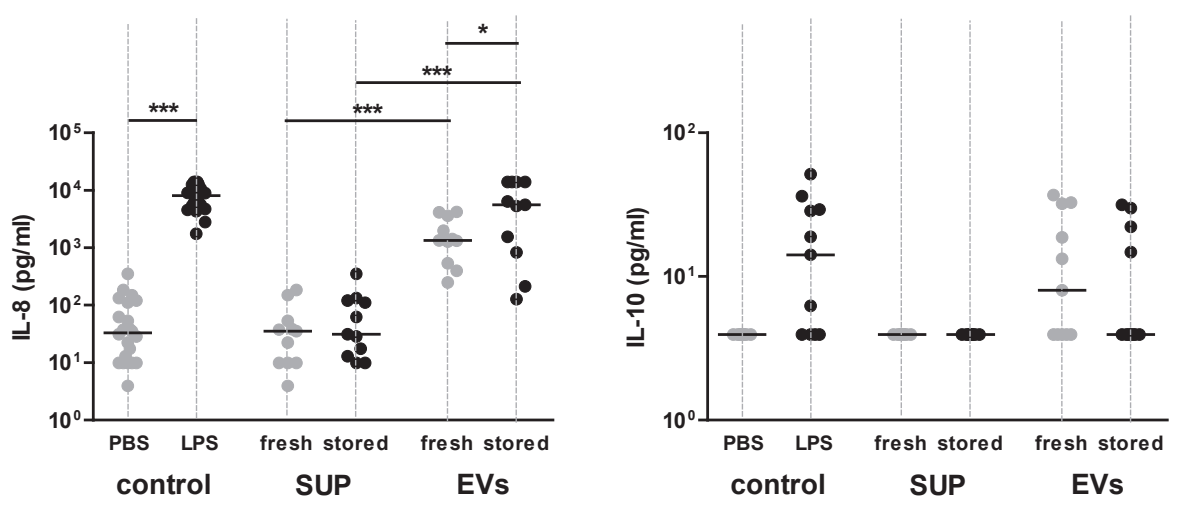

pernatant was isolated and centrifuged again. The EV-containing supernatant was snap frozen and stored at $-80^{\circ} \mathrm{C}$ until further use. Just prior to whole blood stimulation, samples from both fresh ( $\leq 12$ days old) and stored ( $\geq 35$ days old) $\mathrm{RBC}$ bags were thawed and ultracentrifuged for $60 \mathrm{~min}$ at $50,000 \times g, 4{ }^{\circ} \mathrm{C}$. Hereafter, the EV-free supernatant was isolated by pipetting of the upper supernatant fraction. The EV pellet was resuspended in supernatant. EVs were quantified using tunable resistive pulse sensing (TRPS; qNano; Izon Science Ltd, Christchurch, New Zealand), as described by van der Pol et al. [13]. In short, the concentration and size distribution of particles was measured using a NP200A nanopore. This nanopore was suitable for the detection of 100-400 nm particles. Samples were measured with 7 mbar pressure, $45 \mathrm{~mm}$ stretch, and $0.34 \mathrm{~V}$. Samples were analyzed until 1,000 vesicles were counted. To calibrate size and concentration, carboxylated polystyrene beads (Izon Science Ltd) were sonicated for $10 \mathrm{~s}$, diluted in PBS with $0.3 \mathrm{mmol} / \mathrm{l}$ sodium dodecyl sulfate, and analyzed immediately after dilution.

\section{Whole Blood Stimulation}

The response of blood leukocytes to RBC-derived EVs was determined in a whole blood stimulation system. After written informed consent was obtained, blood was drawn from healthy volunteers in $10 \mathrm{ml}$ sodium heparin tubes using the BD Vacutainer system (BD Biosciences, Franklin Lakes, NJ, USA). Immediately after drawing, $250 \mu \mathrm{l}$ of whole blood was 1:1 diluted with RPMI, to which a fixed amount of $50 \mu$ of supernatant from a RBC product was added. Blood was incubated with supernatant from a fresh or stored RBC product, either containing EVs or depleted from EVs. A positive control group was added by stimulation with lipopolysaccharides (LPS) (from Escherichia coli, Sigma St. Louis, MO, USA; $10 \mathrm{ng} / \mathrm{ml}$ ), whereas stimulation with PBS served as a negative control. After $6 \mathrm{~h}$ of incubation at $37^{\circ} \mathrm{C}$ with $5 \% \mathrm{CO}_{2}$, samples were centrifuged at $600 \times \mathrm{g}$ for $10 \mathrm{~min}$ at $4{ }^{\circ} \mathrm{C}$. Supernatant was stored at $-80^{\circ} \mathrm{C}$. Levels of TNF, IL-6, IL-8, and IL-10 were determined by ELISA according to the manufacturer's instructions (R\&D Systems, Minneapolis, MN, USA).

In separate stimulation experiments, the dose response to EVs was investigated. Whole blood from healthy donors $(250 \mu \mathrm{l})$ was stimulated with $250 \mu \mathrm{l}$

supernatant samples from fresh and stored RBC bags, to which fixed consecutive concentrations of EVs were added. EVs were isolated as described above and resuspended in supernatant and diluted with supernatant to a fixed volume containing increasing numbers of EVs.

\section{Statistical Analysis}

Variables are expressed as medians and interquartile ranges. For comparisons, Kruskal-Wallis testing was done followed by Dunn's post test. Statistical significance level of 0.05 was taken. For the analyses, Graphpad Prism 5 (Graphpad Software, San Diego, CA, USA) was used.

\section{Results}

\section{Whole Blood Samples}

We obtained blood from 12 healthy volunteers, aged 24-45 years. Blood groups were mixed (4 times type A, 6 times type B, 2 times type $\mathrm{O}$ ). One volunteer was excluded from analysis because of strongly elevated levels of cytokines in the plasma.

\section{Fresh versus Stored Extracellular Vesicles}

Supernatant from fresh RBC units $(\mathrm{N}=12)$ contained $5.4 \times 10^{6}$ $\pm 1.6 \times 10^{6} / \mu \mathrm{l}$ of EVs, which were around $255.4 \pm 45 \mathrm{~nm}$ in size. The amount of EVs in supernatant from stored RBC units $(\mathrm{N}=12)$ was higher, namely $7.27 \times 10^{7} \pm 1.16 \times 10^{7} / \mu$ l, which were 232.7 $\pm 32 \mathrm{~nm}$ in size. The level of IL- 6 in the supernatant did not differ between fresh $(4.2 \pm 1.4 \mathrm{pg} / \mathrm{ml})$ and stored EV samples $(3.5 \pm 1.1$ $\mathrm{pg} / \mathrm{ml}$; non-significant (NS)), nor the level of TNF (14.2 \pm 7.2 vs. $8.7 \pm 1.9 \mathrm{pg} / \mathrm{ml}$; NS) and the level of IL-8 (3.2 \pm .2 vs. $3.0 \pm 0.1 \mathrm{pg} / \mathrm{ml}$; NS). 

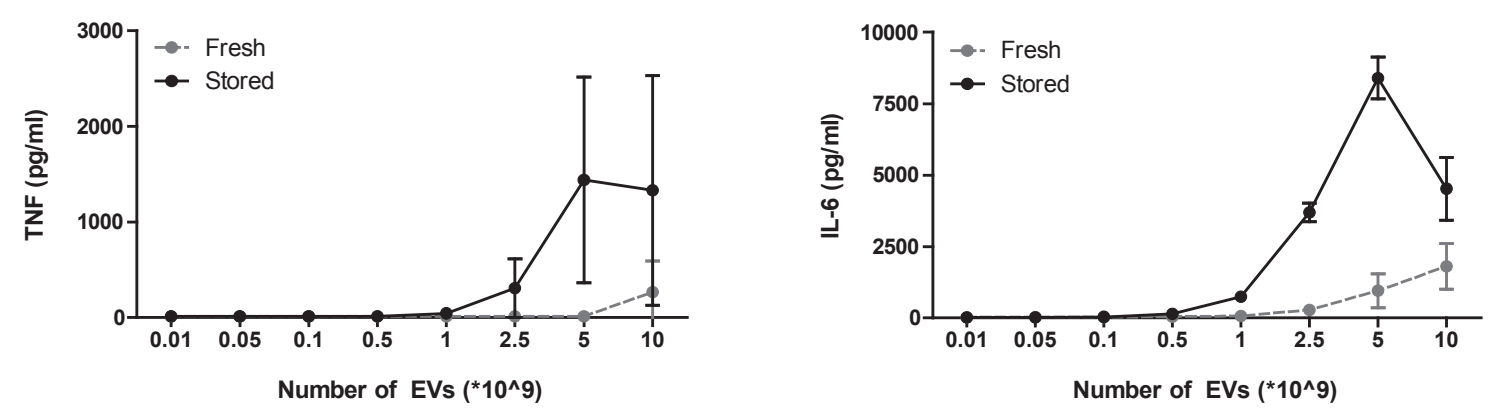

Fig. 2. Whole blood stimulated with supernatant (sup) derived from fresh and stored $\mathrm{RBC}$ bags containing a fixed concentration of EVs.
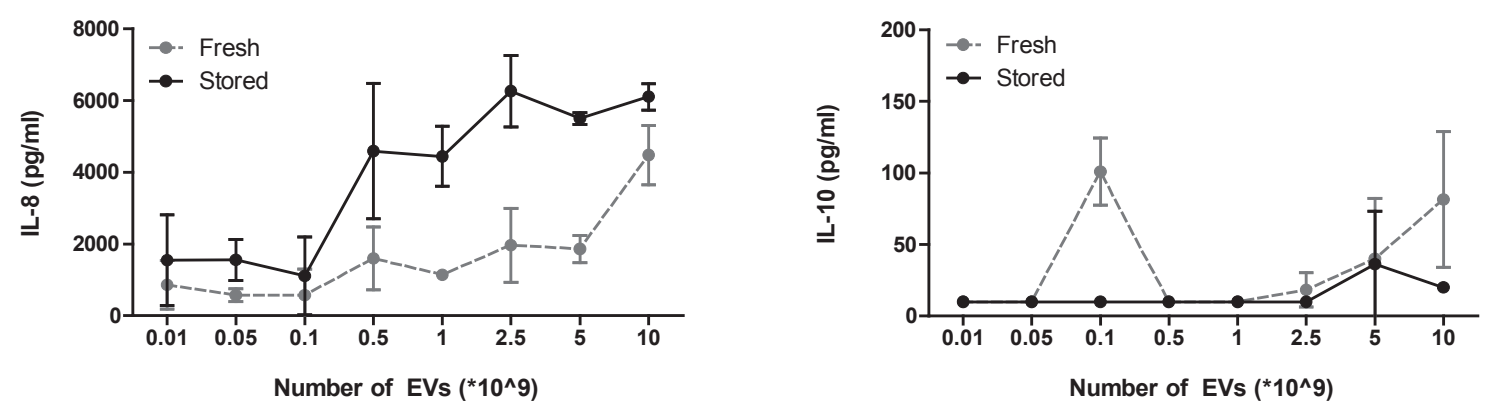

Incubation of whole blood with LPS resulted in increased production of TNF ( $p<0.001)$, IL-6 ( $p<0.001)$, IL-10 ( $p=0.002)$ and IL-8 $(\mathrm{p}<0.001)$ compared to the negative control (fig. 1$)$. The addition of EV-free supernatant from RBC units did not induce a cytokine response compared to the negative control, regardless of storage time. In contrast, the addition of supernatant containing EVs significantly increased levels of all cytokines compared to incubation with supernatant alone. Of note, incubation with stored EVs induced a significantly higher production of TNF $(\mathrm{p}<0.05)$ and IL-6 $(p<0.05)$ compared to incubation with fresh EVs, whereas levels of IL-10 and IL-8 did not differ between fresh and stored EVs (fig. 1).

\section{Concentration Sequence}

After having observed that supernatant with EVs from stored $\mathrm{RBC}$ units induced a stronger host response compared to an equal volume of supernatant with EVs from fresh RBC units, we determined whether the increased response was dependent on the amount of EVs or on alterations in RBC-derived microparticles as a result of storage time. A concentration sequence was performed with supernatant from fresh and stored RBC units with increasing number of EVs. When blood was stimulated with higher numbers of EVs, the production of TNF, IL- 6 and IL- 8 was more pronounced ( $p<0.05$ ) (fig. 2), indicating a dose-response relationship. In contrast, IL-10 production remained low at all numbers of EV. Furthermore, stored EVs induced a higher production of IL-6 and IL-8, but not of TNF, compared to equal amounts of fresh EVs (fig. 2).

\section{Discussion}

Our study shows that RBC-derived EVs induce a strong proinflammatory host response, which in strength equals that of LPS.
In vitro, it was found before that RBC-derived EVs have chemokine binding capacity [14]. Here, we extend these findings with functional studies, showing that RBC-derived EVs can induce inflammation. Of note, a recent study found that both supernatant containing RBC-derived EVs as well as supernatant depleted from EVs have neutrophil priming capacity [15]. The explanation for these different results may be related to differences in processing and storage conditions of RBC units. Indeed, the amount of EVs from leukoreduced RBC units decreased during storage in that study [15], whereas the amount of EVs from leukoreduced RBC units increased during storage in this study. Whether EVs are causal of the adverse effects associated with RBC transfusion in patients is not known, but evidence is emerging that EVs may have an effect on key players during a transfusion-related acute lung injury, including white blood cells and the endothelium. It was recently found that neutrophil-induced activation of pulmonary endothelial cells depended on the presence of plateletderived EVs [16]. Also, in experimental murine models, injection of RBC-derived EVs from stored RBC units was shown to exacerbate lung injury $[17,18]$. In this study, it is not clear whether EVs have a direct effect on host response. In addition to EVs derived from blood bags, endogenous circulating EVs also appear to have pro-inflammatory properties. In an animal model of ischemia reperfusion injury, EVs from endothelium and inflammatory cells are shed into the circulation, which can subsequently promote neutrophil migration [19]. Moreover, we showed in an earlier investigation that in trauma patients endogenously circulating EVs can induce an inflammatory host response [20]. As the EVs in this study are derived from allogenic blood, inflammation may have been induced allogenously, but the EVs may also have caused an endogenous host response. The exact mechanism remains to be determined. 
In this study, the pro-inflammatory capacity of RBC-derived EVs is dose-dependent. As numbers of EVs increased during storage, EVs may be considered as a part of the RBC storage lesion. Given that incubation of EVs, but not the stored supernatant, induced inflammation, we think that EVs and not another soluble factor in the supernatant are causative in mediating host response.

Dose-dependent activation of the host response was also present with EVs derived from fresh RBC units, suggesting that EVs are pro-inflammatory regardless of storage time. This finding may have clinical relevance. If EVs from RBCs are related to adverse effects of transfusion, interventions aimed at decreasing EVs prior to transfusion, such as use of a cell saver, may improve outcome of RBC transfusion.

In conclusion, this study shows that EVs from both fresh and stored RBC bags illicit a strong inflammatory host response in recipients. This response depends both on the number of EVs as well as on changes of the EVs related to storage.

\section{Disclosure Statement}

The authors declare that they have no competing interests

\section{References}

1 Marik PE, Corwin HL: Efficacy of red blood cell transfusion in the critically ill: a systematic review of the literature. Crit Care Med 2008;36:2667-2674.

2 Ferraris VA, Ballert EQ, Mahan A: The relationship between intraoperative blood transfusion and postoperative systemic inflammatory response syndrome. Am J Surg 2013;205:457-465.

3 Rubinstein C, Davenport DL, Dunnagan R, Saha SP Ferraris VA, Xenos ES: Intraoperative blood transfusion of one or two units of packed red blood cells is associated with a fivefold risk of stroke in patients undergoing elective carotid endarterectomy. J Vasc Surg 2013;57(2 suppl):53S-57S

4 Hod EA, Zhang N, Sokol SA, Wojczyk BS, Francis RO, Ansaldi D, Francis KP, Della-Latta P, Whittier S, Sheth S, Hendrickson JE, Zimring JC, Brittenham GM, Spitalnik SL: Transfusion of red blood cells after prolonged storage produces harmful effects that are mediated by iron and inflammation. Blood 2010;115:42844292.

5 Vlaar AP, Hofstra JJ, Levi M, Kulik W, Nieuwland R, Tool AT, Schultz MJ, de Korte D, Juffermans NP: Supernatant of aged erythrocytes causes lung inflammation and coagulopathy in a 'two-hit' in vivo syngeneic transfusion model. Anesthesiology 2010;113:92-103.

6 Fergusson DA, Hebert P, Hogan DL, LeBel L, Rouvinez-Bouali N, Smyth JA, Sankaran K, Tinmouth A, Blajchman MA, Kovacs L, Lachance C, Lee S, Walker CR, Hutton B, Ducharme R, Balchin K, Ramsay T, Ford JC, Kakadekar A, Ramesh K, Shapiro S: Effect of fresh red blood cell transfusions on clinical outcomes in premature, very low-birth-weight infants: the ARIPI randomized trial. JAMA 2012;308:1443-1451.
7 Koch CG, Li L, Sessler DI, Figueroa P, Hoeltge GA, Mihaljevic T, Blackstone EH: Duration of red-cell storage and complications after cardiac surgery. N Engl J Med 2008;358:1229-1239.

8 Lacroix J, Hebert PC, Fergusson DA, Tinmouth A, Cook DJ, Marshall JC, Clayton L, McIntyre L, Callum J, Turgeon AF, Blajchman MA, Walsh TS, Stanworth SJ, Campbell H, Capellier G, Tiberghien P, Bardiaux L, van de Watering L, van der Meer NJ, Sabri E, Vo D; ABLE Investigators; Canadian Critical Care Trials Group: Age of transfused blood in critically ill adults. N Engl J Med 2015;372:1410-1418.

-9 Yuana Y, Sturk A, Nieuwland R: Extracellular vesicles in physiological and pathological conditions. Blood Rev 2013;27:31-39.

10 Rubin O, Delobel J, Prudent M, Lion N, Kohl K, Tucker EI, Tissot JD, Angelillo-Scherrer A: Red blood cell-derived microparticles isolated from blood units initiate and propagate thrombin generation. Transfusion 2013;53:1744-1754.

11 Gao Y, Lv L, Liu S, Ma G, Su Y: Elevated levels of thrombin-generating microparticles in stored red blood cells. Vox Sang 2013;105:11-17.

12 Jy W, Ricci M, Shariatmadar S, Gomez-Marin O, Horstman LH, Ahn YS: Microparticles in stored red blood cells as potential mediators of transfusion complications. Transfusion 2011;51:886-893.

13 van der Pol E, Coumans FA, Grootemaat AE, Gardiner C, Sargent IL, Harrison P, Sturk A, van Leeuwen TG, Nieuwland R: Particle size distribution of exosomes and microvesicles determined by transmission electron microscopy, flow cytometry, nanoparticle tracking analysis, and resistive pulse sensing. J Thromb Haemost 2014;12:1182-1192.
14 Xiong Z, Cavaretta J, Qu L, Stolz DB, Triulzi D, Lee JS Red blood cell microparticles show altered inflammatory chemokine binding and release ligand upon interaction with platelets. Transfusion 2011;51:610-621.

15 Kent MW, Kelher MR, West FB, Silliman CC: The proinflammatory potential of microparticles in red blood cell units. Transfus Med 2014:24:176-181.

16 Xie RF, Hu P, Wang ZC, Yang J, Yang YM, Gao L, Fan $\mathrm{HH}$, Zhu YM: Platelet-derived microparticles induce polymorphonuclear leukocyte-mediated damage of human pulmonary microvascular endothelial cells. Transfusion 2015;55:1051-1057.

17 Belizaire RM, Prakash PS, Richter JR, Robinson BR, Edwards MJ, Caldwell CC, Lentsch AB, Pritts TA: Microparticles from stored red blood cells activate neutrophils and cause lung injury after hemorrhage and resuscitation. J Am Coll Surg 2012;214:648-655; discussion 656-647.

18 Zecher D, Cumpelik A, Schifferli JA: Erythrocyte-derived microvesicles amplify systemic inflammation by thrombin-dependent activation of complement. Arterioscler Thromb Vasc Biol 2014;34:313-320.

19 Teoh NC, Ajamieh H, Wong HJ, Croft K, Mori T, Allison AC, Farrell GC: Microparticles mediate hepatic ischemia-reperfusion injury and are the targets of Diannexin (ASP8597). PLoS One 2014;9:e104376.

20 Balvers K, Curry N, Kleinveld DJ, Boing AN, Nieuwland R, Goslings JC, Juffermans NP: Endogenous microparticles drive the proinflammatory host immune response in severely injured trauma patients. Shock 2015;43:317-321. 